\title{
Effects of a Specific Workshop on the Beliefs and Perceptions on Zoonosis of the Veterinary Students: A First Pilot Longitudinal Study
}

\begin{abstract}
Véronique Renault ${ }^{1}$, Sébastien Fontaine ${ }^{2}$ and Claude Saegerman ${ }^{3 *}$
${ }^{1}$ Research Unit in Epidemiology and Risk Analysis Applied to Veterinary Sciences (UREAR-ULiege), Fundamental and Applied Research for Animal Health (FARAH) Centre, Faculty of Veterinary Medicine, University of Liege, Belgium

${ }^{2}$ Research Institute in Social Sciences, Department of Social Sciences, Faculty of Social Sciences, University of Liege, Belgium

${ }^{3}$ Research Unit in Epidemiology and Risk Analysis Applied to Veterinary Sciences (UREAR-ULiege), Fundamental and Applied Research for Animal Health (FARAH) Centre, Faculty of Veterinary Medicine, University of Liege, Belgium

*Corresponding author: Claude Saegerman, Research Unit in Epidemiology and Risk Analysis Applied to Veterinary Sciences (UREAR-ULiege), Fundamental and Applied Research for Animal Health (FARAH) Centre, Faculty of Veterinary Medicine, University of Liege, Belgium
\end{abstract}

\section{ARTICLE INFO}

Received: 幽 April 20, 2021

Published: 巣 April 29, 2021

Citation: Renault V, Fontaine S, Saegerman C. Effects of a Specific Workshop on the Beliefs and Perceptions on Zoonosis of the Veterinary Students: A First Pilot Longitudinal Study. Biomed J Sci \& Tech Res 35(3)2021. BJSTR. MS.ID.005702.

Keywords: One Health; Biosecurity; Veterinarians; Health Belief Model; Perception; Risk; Behavior

\section{ABSTRACT}

Background: Zoonoses represent a major threat and an occupational health hazard for the veterinary practitioners. Their prevention relies mainly on the implementation of different biosecurity measures (BSM). Nevertheless, the existing studies demonstrate a low level of implementation of BSM by the veterinary professionals. The objective of this study is to assess the level of implementation or intention to implement the BSM by the veterinary students before and after a specific workshop on zoonosis in order to evaluate the efficacy of such training activities on the adoption of the proper behaviour. Methods and principal findings: The data was collected through a longitudinal survey $(\mathrm{N}=41)$. The different beliefs and perceptions of the respondents as well as their level of implementation or intention to implement the different biosecurity measures were assessed before and after the survey and compared in order to evaluate the possible effect of the workshop on these different elements.

Conclusion and Significance: The workshop did not have any significant influence on the intention to adopt a proper behaviour. In the future, it is recommended to increase the duration of training sessions on this topic throughout the cursus as well as to pursue such communications towards the veterinary professionals.

\section{Short Communication}

Zoonoses represent $60 \%$ of the diseases affecting humans and $75 \%$ of emerging diseases [1]. They represent a major threat in public health and their socio-economic impact are important as illustrated by the 2020 pandemic of COVID-19 which has severely affected the economy and the social lives of the population worldwide. Zoonoses prevention relies mainly on biosecurity measures which should be emphasized at the interface of animals, humans and environment. The veterinary professionals are more likely to be exposed to these pathogen agents [2] and the veterinary students appear to be exposed early in their education
$[3,4]$. Despite these findings, different studies reported a generally low level of implementation of the biosecurity measures (BSM) by the veterinary professionals $[5,6]$. According to the Health Belief Model (HBM), the intention to implement a given behaviour is determined by 5 mental constructs:

a. The risk susceptibility (perceived likelihood of the risk occurrence),

b. The risk severity (perceived impact of the risk if it occurs),

c. The benefits of the behaviour, 
d. The barriers to the behaviour implementation or outcomes

e. The health responsibility (perceived responsibility towards animal, public and environmental health) [7].

These constructs can be influenced through proper communication and awareness raising. Nevertheless, the training received in terms of zoonotic risks and biosecurity is judged insufficient by $28 \%$ of the students at the Faculty of Veterinary Medicine of Liege (Renault V., 2020, unpublished data). Such training sessions could easily be developed and included in the professional training of the veterinary students. The objective of this pilot study was to assess the actual impact of a two-hours workshop on the zoonotic risks on the different constructs of the HBM and on the intention of the veterinary students to implement the BSM in their future practices.

\section{Materials and Methods}

\section{Survey Design and Implementation}

In order to raise awareness of the veterinary students on the zoonotic risks and the biosecurity measures to implement, a specific workshop on zoonosis was developed. It was designed as case studies to be presented by the student on different zoonotic diseases affecting different species in order to promote self-reflection and exchanges among small groups of 10 to 16 students from end of September 2019 to April 2020. A longitudinal study was carried on and the data was collected by an on-line survey developed with Lime Survey, an open-source web application. The questionnaire was directed to veterinary students in Master two or three (Appen$\operatorname{dix}$ A) registered for the paraclinics seminars under which the workshop on zoonoses was to be delivered. The students' survey

Table 1: List of statements used to assess the different Health Belief Model constructs by asking the respondent their degree of agreement through a visual analogue scale [8].

\begin{tabular}{|c|c|}
\hline HBM construct & Statements used for the Indirect Assessment of the Construct \\
\hline \multirow{4}{*}{ Susceptibility } & According to me, veterinary practitioners are very frequently exposed to zoonotic infectious diseases \\
\hline & According to me, zoonotic infectious diseases represent a major risk for veterinary practitioners \\
\hline & $\begin{array}{l}\text { As a veterinary practitioner, I could easily and unwillingly be responsible of the spread of a zoonotic disease to my relatives or to } \\
\text { other persons }\end{array}$ \\
\hline & My future professional practice represents an important risk for my health \\
\hline \multirow{3}{*}{ Severity } & If I were to contract a major zoonotic disease, my incomes would be heavily impacted \\
\hline & If I were to contract a major zoonotic disease, my life quality would be severely affected \\
\hline & If I were to contract a major zoonotic disease, I might contaminate my relatives and other persons \\
\hline \multirow{6}{*}{ Benefits } & $\begin{array}{l}\text { According to you, what is the efficiency of the following biosecurity measures to prevent yourself from a possible contamination ( } 0 \text { : } \\
\text { useless, 100: very effective (full protection): }\end{array}$ \\
\hline & BSM0. The different preventive measures which can be taken by the veterinarians \\
\hline & BSM 1. Disinfecting your hands after each manipulation (or cleaning them with an antibacterial soap or solution) \\
\hline & BSM 2. Inquiring from the owner about the country of origin of the animal in consultation \\
\hline & BSM 3. Protecting my hands by wearing gloves adapted to the need(s) \\
\hline & $\begin{array}{l}\text { BSM 4. Protecting myself from oro-nasal contaminations by wearing a mask in case of interventions likely to cause projections (e.g. } \\
\text { abscess puncture, wound cleaning, descaling, autopsy) }\end{array}$ \\
\hline
\end{tabular}

was conducted twice: once before the workshops (from September 2019 up to end of May 2020) and a second time at the end of the academic year (from June up to end of July 2020). The invitations to fill in the first survey were sent on a monthly basis to the students through the mailing lists of the Students' Office after approval of the Dean of the Faculty of Veterinary Medicine. Specific reminders were also sent to the different student groups about 10 days before their workshop on the zoonosis in order to increase the answer rate for the first survey. For the second survey, the invitations were sent in May, June and July (just after the examination session).

In the first survey, different questions were asked to assess the different components of the different HBM constructs and the level of implementation of the BSMs by the respondent [8]. Existing HBM guidelines [9,10] and questionnaires [11-15] were used in order to develop the different questions related to the HBM constructs which were mainly assessed indirectly by asking the respondents their degree of agreement (0: fully disagree and 100: fully agree) with different statements at the exception of the perceived benefits of the different BSMs which were assessed by a direct question [8]. The second survey included only the questions related to the HBM constructs as well as some questions related to the eventual practical experience acquired in between (Table 1). The construct called 'health responsibility' was not assessed and compared as the workshop emphasized mainly on the zoonotic risk and their preventive measures. No effect on the 'Health responsibility' perception was therefore expected. The student's identification number was used to pair the data and to control that the student did benefits from the workshop on zoonosis between the 2 surveys and to analyze whether the training workshops that they attended did change their beliefs and perceptions regarding the zoonotic risks and the ways to prevent them. 


\begin{tabular}{|c|c|}
\hline & $\begin{array}{l}\text { BSM 5. Protecting myself against ocular contaminations by wearing protective glasses during interventions likely to cause } \\
\text { projections (e.g. descaling, autopsy) }\end{array}$ \\
\hline & BSM 6. Throwing the needles directly into a specific container without replacing the cap \\
\hline & BSM 7. Washing dirty clothing separately with a proper cleaning cycle \\
\hline & BSM 8. Being vaccinated against rabies \\
\hline & BSM 9. Ensuring a proper contention in order to avoid being wounded (bites, scratches ,..) \\
\hline & $\begin{array}{c}\text { BSM 10. In case of wound (bites, scratches ,..), proceeding to the immediate cleaning with an antiseptic soap or solution (a few } \\
\text { minutes after the event maximum) }\end{array}$ \\
\hline & BSM 11. Keeping myself updated of the new evolutions in terms of zoonoses and their prevention (continuous training) \\
\hline & BSM 12. Using disposable coat for single use \\
\hline & BSM 13. Cleaning my boots when exiting the holdings \\
\hline \multirow{3}{*}{ Barriers } & No measure is really effective; I am exposed to zoonotic infections anyway \\
\hline & By my practices, I am able to considerably lower the exposure and contamination risks to a zoonotic disease \\
\hline & $\begin{array}{l}\text { Performing hygienic measures (e.g. hands, boots) is only possible if the holdings are equipped with proper cleaning infrastructures. } \\
\text { If there are no cleaning spots on the holdings, we cannot perform these measures }\end{array}$ \\
\hline
\end{tabular}

\section{Statistical Analysis}

The data originating from completed questionnaires were extracted to Microsoft Excel(C) and the responses given by the participants were coded in accordance with Appendix 1. The longitudinal study only included the students who did answered to the first survey prior to the workshop, attended the workshop and answered the second survey after the workshop. The representability of the sample used in the longitudinal study to compare the perceptions before and after the workshop was tested by comparing the gender, year of education and practical experiences of the respondents compared to the general master student population. The different comparisons were based on a chi-square test performed in Stata SE/14. The scoring of the four HBM constructs was done as described in a previous study which analysed the determining factors of the biosecurity measures by the veterinary professionals based on the results of the first survey [8]. The perceived benefits of BSMs were assessed through a single question generating a BSM efficiency score ranging from 0 to 100 . The average score of all the BSM efficiency score (13 in total) was also calculated and called "Overall score" for the benefits' perceptions. The other HBM constructs, were determined indirectly by asking a set of questions or items. The scores of each construct were calculated as the mean score of each of the items and range from 0 to 100 . The level of intention to implement a BSM or the actual level of implementation were graded from 0 (Never implemented) to 4 (Always implemented) and an overall BS score was calculated by estimating the overall level of implementation of the individual BSM in percentage of the maximum score possible obtained (if all the BSM are always implemented). The perception scores related to the risk susceptibility, risk severity, barriers and benefits as well as the different BS implementation scores were calculated in the first and second survey and paired based on the student's identification number. The perceptions before and after the workshop were then compared by a Wilcoxon signed-rank test (for paired data) using Stata SE/14.

\section{Results}

The longitudinal study was implemented as planned in order to capture the student beliefs and perceptions before the workshop. Nevertheless, the COVID 19 pandemic interrupted the workshops on March 2020 (Figure 1). The students' answer rates in the first and second survey were of 35 and 44\%, respectively. The answer rate of the second survey was calculated as a percentage of the students who responded to the second survey among the 181 students who agreed to participate in the longitudinal study. However, due to the COVID 19 outbreak and resulting confinement from March $15^{\text {th }}$ up to the end of the academic year, only 41 of the 77 respondents of the second survey benefitted from the workshop on zoonoses. Therefore, the students for which the perceptions could be compared only represented $18 \%$ of the students who completed the first survey. The chi-square test to compare the proportion of men and women in the overall population of students and the survey populations did not demonstrate any statistical differences ( $p>0.05$ ). Based on the same analysis, no statistical difference was found when comparing the students' experiences in the different kind of practices. When considering the 41 respondents included in the longitudinal study, $87.8 \%$ of the respondents are female and $97.6 \%$ of them reported having a practical experience through various internships (Table 2). The perceived susceptibility and barriers were significantly lower after the workshop (p-values of 0.03 and 0.005 , respectively) while no significant differences were observed for the other HBM constructs (Figure 2). 


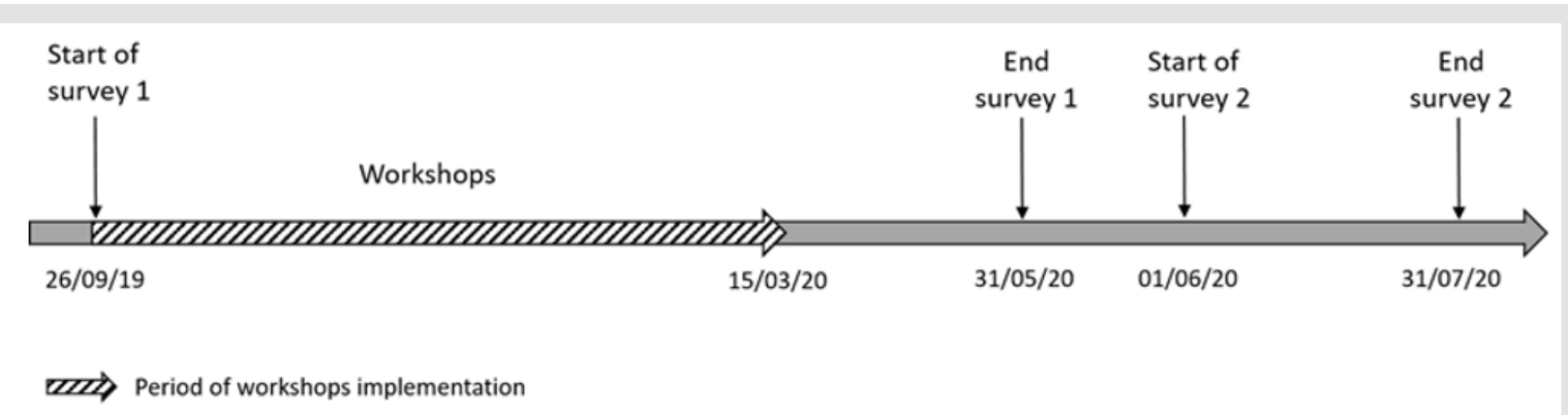

Figure 1: Timeline of the longitudinal survey.

[A] Susceptibility*

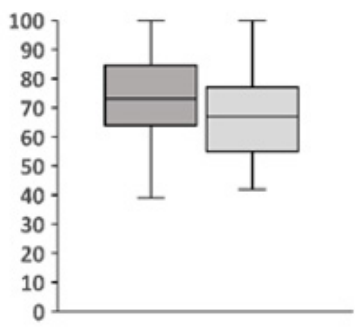

[D] Benefits

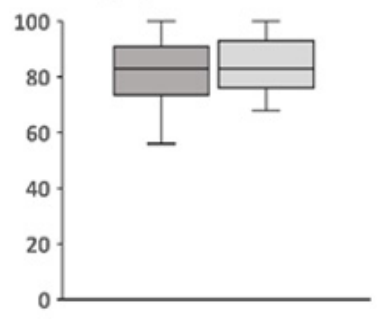

[B] Severity

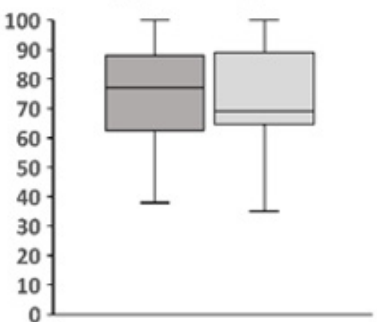

[E] Biosecurity Score

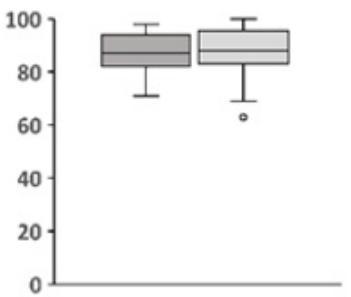

[C] Barriers*

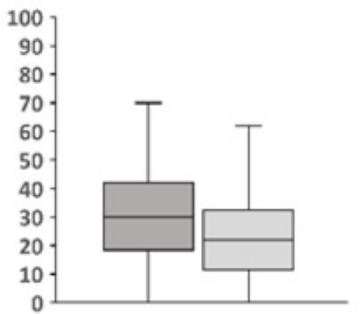

Figure 2: Perception scores of the Health Belief Model constructs.

Note: * Percetpion scores significantly different at the beginning and at the end of the survey period.

Table 2: Demographics of the respondents.

\begin{tabular}{|c|c|c|c|c|c|c|c|c|}
\hline \multirow[b]{2}{*}{ Respondents } & \multirow[b]{2}{*}{ Year of Education } & \multirow[b]{2}{*}{$\mathbf{N}$} & \multicolumn{2}{|c|}{ Gender } & \multirow[b]{2}{*}{ None/ Other } & \multicolumn{3}{|c|}{ Kind of Practice } \\
\hline & & & Female & Male & & $\begin{array}{c}\text { Large } \\
\text { Animals }\end{array}$ & $\begin{array}{c}\text { Small } \\
\text { Animals }\end{array}$ & Mixed \\
\hline \multirow{3}{*}{$\begin{array}{l}\text { Veterinary students } \\
\text { (Survey } 1 \text { ) }\end{array}$} & Total of students & 227 & $78.41 \%$ & $21.59 \%$ & $7.49 \%$ & $3.96 \%$ & $48.90 \%$ & $39.65 \%$ \\
\hline & Master 2 & 162 & $75.31 \%$ & $24.69 \%$ & $7.05 \%$ & $2.20 \%$ & $35.68 \%$ & $26.43 \%$ \\
\hline & Master 3 & 65 & $86.15 \%$ & $13.85 \%$ & $1.54 \%$ & $6.15 \%$ & $46.15 \%$ & $46.15 \%$ \\
\hline \multirow{3}{*}{$\begin{array}{l}\text { Veterinary students } \\
\text { (Survey 2) }\end{array}$} & Total of students & 78 & $79.49 \%$ & $20.51 \%$ & $3.85 \%$ & $6.41 \%$ & $48.72 \%$ & $41.03 \%$ \\
\hline & Master 2 & 54 & $75.93 \%$ & $24.07 \%$ & $5.56 \%$ & $5.56 \%$ & $50.00 \%$ & $38.89 \%$ \\
\hline & Master 3 & 24 & $87.50 \%$ & $12.50 \%$ & $0.00 \%$ & $8.33 \%$ & $45.83 \%$ & $45.83 \%$ \\
\hline \multirow{3}{*}{ Longitudinal Study } & Total of students & 41 & $87.80 \%$ & $12.20 \%$ & $2.44 \%$ & $4.88 \%$ & $46.34 \%$ & $46.34 \%$ \\
\hline & Master 2 & 24 & $83.33 \%$ & $16.67 \%$ & $4.17 \%$ & $4.17 \%$ & $45.83 \%$ & $45.83 \%$ \\
\hline & Master 3 & 17 & $94.12 \%$ & $5.88 \%$ & $0.00 \%$ & $5.88 \%$ & $47.06 \%$ & $47.06 \%$ \\
\hline
\end{tabular}




\section{Discussion}

This is the first pilot study investigating the possible effect of an intervention on the different beliefs and perceptions, which influences the implementation of preventive measures against zoonoses. The results are useful to determine if the students are sufficiently prepared to properly identify and address the zoonotic risks in their future practice in order to better preserve their health as well as the animals, humans and environmental health. The survey methodology addressed the possible volunteer bias by sending several reminders in order to increase the answer rate and managed an answer rate of $35 \%$ for the first survey with $44 \%$ of the respondents answering to the second survey. Such rates seem to be acceptable as personalized internet surveys usually generate a response rate of $4.7 \%$ [16]. Unfortunately, due to the COVID pandemic and despite a proper answer rate to the students' second survey, many students were not able to benefit from the training workshop on zoonoses and had to be excluded from the analysis. This affected the number of students eligible to the longitudinal study. Nevertheless, the sample can be considered representative of the overall population of students as the proportions of males and females as well as the proportions of mixed, small animals and rural practitioners were not significantly different among the groups. The workshop on zoonoses which was implemented during the academic year in order to raise awareness among the students on the zoonotic risks in veterinary practices and the importance of implementing proper BSM in order to facilitate the adoption of these measures by the student in their professional practices. In regards to the perceptions, the workshop was expected to increase the students' perceptions of the zoonotic risks and BSMs' benefits while reducing the barriers' perceptions.

If the barriers' perceptions is indeed significantly lower at the end of the year, the students' perception of the zoonoses' susceptibility was significantly lower at the end of the year (after attending the workshop) which is supposed to negatively affect the level of implementation of the BSMs. Many reasons could explain such observations, one of them being a failure of the intervention to change the behaviour as it has been the case in other interventional studies under which a more intensive communication on behaviour change was conducted [17]. It would illustrate the need to improve the communication campaigns by identifying the determinant factors and using them to trigger the proper behaviour. However, other elements might have influenced the perceptions as well, and interfered with the actual behaviour (e.g. internships and personal experiences). Out of the 41 respondents, $37 \%$ of them did made an internship between the first and the second survey and the behaviors and attitude of their supervisor regarding BSM might have an important influence on their perceptions. Considering that the level of implementation of some BSM by the veterinary practitioners are generally low as well as their risk perceptions [6], they might have a negative impact of the students beliefs, perceptions and practices.
The survey methodology for this longitudinal study was to assess the students' perceptions before the workshop and at the end of the academic year, not directly after the workshop in order to better measure the long-term impact of the workshop, leaving enough time for other external events to either consolidate or reverse the possible changes in beliefs and attitudes.

It would have been interesting to monitor the changes of perceptions and behaviors throughout the year instead of running only two surveys (one at the beginning and one at the end) in order to better capture the eventual changes over time, their duration and the events which could have determined these changes (e.g. internships). It also appear that a 2 hours workshop although judged really interesting and bringing a real added value (unpublished data on the students' feed-back) might be insufficient and that additional training activities on the topic should be integrated to the veterinary training in the future. As the analysis of the outcomes of the first survey, showed that the BS score was mainly influenced by the zoonoses susceptibility and BSM benefits, the communication materials should emphasize on these two aspects [8]. In addition, the information provided to the students and veterinary professionals should rely on evidence based studies as it appears that most of the time, the decision of the veterinary professionals to either implement or not a BSM is based on the case specific perception of the risks related to the intervention to be performed [8].

\section{Conclusion}

The level of implementation of the BSMs aiming at preventing zoonotic diseases by the veterinary practitioners in Belgium should be increased. One of the main strategies identified relies on a better training of the veterinary student on theses aspects. Nevertheless, a 2 hours workshop on the topic did not seem to have any significant influence and was proven inefficient even on a short-term basis. As for most interventional activities aiming at a long-term behaviour change, it is therefore recommended to increase the training sessions on this topic throughout the course as well as to pursue such communications towards the veterinary professionals in the future.

\section{Author Contributions}

Conceptualization, Véronique Renault and Claude Saegerman; methodology, Véronique Renault; software, Véronique Renault.; validation, Claude Saegerman and Sebastien Fontaine; formal analysis, Véronique Renault; investigation, Véronique Renault; resources, Véronique Renault; data curation, Véronique Renault; writing - original draft preparation, Véronique Renault; writtingreview and editing, Claude Saegerman and Sebastien Fontaine; visualization, Véronique Renault; supervision, Claude Saegerman and Sebastien Fontaine; project administration, Claude Saegerman. All authors have read and agreed to the published version of the manuscript. 


\section{Funding}

This research received no external funding.

\section{Informed Consent Statement}

Informed consent was obtained from all subjects involved in the study. The data was collected, stored and used in accordance with the GDPR (EU) 2016/679.

\section{Acknowledgment}

We would like to thank all students that participated to the study.

\section{Conflicts of Interest}

The authors declare no conflict of interest.

\section{References}

1. Taylor LH, Latham SM, Woolhouse ME (2001) Risk factors for human disease emergence. Philos Trans R Soc Lond B Biol Sci 356(1411): 983989.

2. Baker WS, Gray GC (2009) A review of published reports regarding zoonotic pathogen infection in veterinarians. J Am Vet Med Assoc 234(10): 1271-1278.

3. De Rooij MMT, Schimmer B, Versteeg B, Schneeberger P, Berends BR, et al. (2012) Risk Factors of Coxiella burnetii (Q Fever) Seropositivity in Veterinary Medicine Students. PLoS One 7(2): e32108.

4. Sánchez A, Pratsvan der Ham M, Tatay Dualde J, Paterna A, De la Fe C, et al. (2017) Zoonoses in Veterinary Students: A Systematic Review of the Literature. PLoS One 12: e0169534.

5. Sayers RG, Good M, Sayers GP (2014) A survey of biosecurity-related practices, opinions and communications across dairy farm veterinarians and advisors. Vet J 200(2): 261-269.

6. Renault V, Humblet MF, Moons V, Bosquet G, Gauthier B, et al. (2018) Rural veterinarian's perception and practices in terms of biosecurity

\section{ISSN: 2574-1241}

\section{DOI: 10.26717/BJSTR.2021.35.005702}

Claude Saegerman. Biomed J Sci \& Tech Res

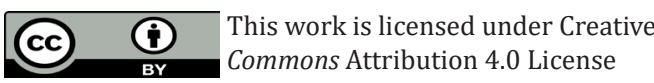

Submission Link: https://biomedres.us/submit-manuscript.php across three European countries. Transbound Emerg Dis 65(1): e183-e193.

7. Abraham C, Sheeran P (2015) The Health Belief Model. In: Mark Conner, Paul Norman (Eds.)., Maidenhead, p. 30-69.

8. Renault V, Fontaine S, Saegerman C (2021) Factors Determining the Implementation of Measures Aimed at Preventing Zoonotic Diseases in Veterinary Practices. Pathogens 10(4): 436.

9. Champion VL (1984) Instrument development for health belief model constructs. ANS Adv Nurs Sci 6(3): 73-85.

10. Cummings KM, Jette AM, Rosenstock IM (1978) Construct validation of the health belief model. Health Educ. Monogr 6(4): 394-405.

11. Brennan M, Wright N, Wapenaar W, Jarratt S, Hobson West P, et al. (2016) Exploring Attitudes and Beliefs towards Implementing Cattle Disease Prevention and Control Measures: A Qualitative Study with Dairy Farmers in Great Britain. Animals 6(10): 61.

12. Mankad A (2016) Psychological influences on biosecurity control and farmer decision-making. A review. Agron Sustain Dev 36: 1-14.

13. Ritter C, Jansen J, Roche S, Kelton DF, Adams CL, et al. (2017) Invited review: Determinants of farmers' adoption of management-based strategies for infectious disease prevention and control. J Dairy Sci 100(5): 3329-3347.

14. Vande Velde F, Charlier J, Hudders L, Cauberghe V, Claerebout E (2018) Beliefs, intentions, and beyond: A qualitative study on the adoption of sustainable gastrointestinal nematode control practices in Flanders' dairy industry. Prev Vet Med 153: 15-23.

15. Vande Velde F, Claerebout E, Cauberghe V, Hudders L, Van Loo H, et al. (2015) Diagnosis before treatment: Identifying dairy farmers' determinants for the adoption of sustainable practices in gastrointestinal nematode control. Vet Parasitol 212(3-4): 308-317.

16. Sinclair M, O Toole J, Malawaraarachchi M, Leder K (2012) Comparison of response rates and cost-effectiveness for a community-based survey: postal, internet and telephone modes with generic or personalized recruitment approaches. BMC Med Res Methodol 12: 132.

17. Willemsen A, Cobbold R, Gibson J, Wilks K, Lawler S, et al. (2019) Infection control practices employed within small animal veterinary practices-A systematic review. Zoonoses Public Health 66(5): 439-457.

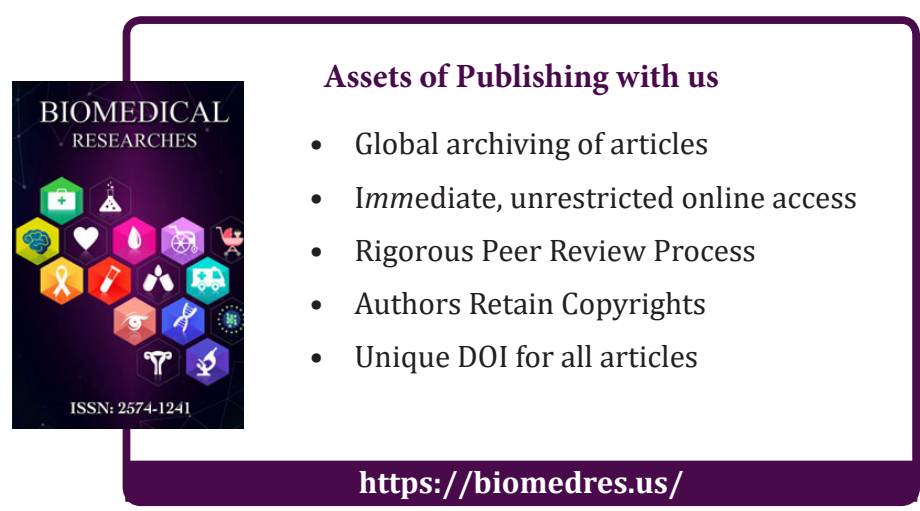

